\title{
The application of design criteria for locating a hub configured supply chain for a restaurant cluster in the Stellenbosch area
}

\author{
Authors: \\ Claudia B. Struwig \\ George A. Ruthven ${ }^{1}$ \\ Konrad von Leipzig ${ }^{1}$ \\ Affiliations: \\ ${ }^{1}$ Department of Industrial \\ Engineering, Stellenbosch \\ University, South Africa \\ Correspondence to: \\ George Ruthven \\ Email: \\ gar@sun.ac.za \\ Postal address: \\ Private Bag X1, Matieland \\ 7602 , South Africa \\ Dates: \\ Received: 08 Jan. 2013 \\ Accepted: 03 May 2013 \\ Published: 25 June 2013 \\ How to cite this article: \\ Struwig, C.B., Ruthven, G.A. \\ \& Von Leipzig, K., 2013, \\ 'The application of design \\ criteria for locating a hub \\ configured supply chain for \\ a restaurant cluster in the \\ Stellenbosch area', Journal \\ of Transport and Supply \\ Chain Management 7(1), Art. \\ \#90, 7 pages. http://dx.doi. \\ org/10.4102/jtscm.v7i1.90

\section{Copyright:} \\ (C) 2013. The Authors. \\ Licensee: AOSIS \\ OpenJournals. This work \\ is licensed under the \\ Creative Commons \\ Attribution License.
}

Read online:
Restaurants, in general, utilise numerous suppliers. Normally they deliver on different days in the week and at different times during the day, logistically not an optimum approach. Not only does the current practice cause frequent interruptions, but by segregating the food supply chain unnecessary traffic is generated. This article investigated the need for developing a third party supplier hub, the best positioning of that hub and the most economical routes to the customers. With the aim of providing non-franchised restaurants with the necessary leverage to become market leaders, the hub is planned to only service the restaurants within the vicinity of the Stellenbosch area. In such a hub-configured supply chain, the suppliers would be delivering to the proposed hub, from where once-off deliveries to all the restaurants may be made on days and/or times determined by them. In order to investigate the benefits of providing such a hub structure, a systematic implementation approach was used. The first step was to do market research in order to establish the need for such a hub. That is, the viability of the hub from a restaurant's perspective was established. The next step entailed the investigation of the design criteria needed in determining a favourable hub location. Four possible hub locations were identified. The Clark and Wright's savings algorithm was then used to determine the optimal hub location and the feasibility of the results was verified with the aid of a global positioning system (GPS) device. The last steps followed involved the determination of an effective hub floor plan that may be utilised, possible products that can be supplied to the restaurants and the necessary assets needed to provide the hub's service. Finally, by incorporating all these facets, a cost analysis was done to determine the hub's profitability.

\section{Introduction}

\section{Background}

\section{The Stellenbosch town and university}

Excluding the students enrolled at the University, the town Stellenbosch has a population of well over 130000 people (Community Connections Online 2011). According to the annual official Stellenbosch University (SU) census of June 2012, the University had a total of 27823 students in 2012. Not only does SU have an active international division, but the town Stellenbosch is also a very popular tourist attraction. Stellenbosch is a vibrant town that has many road-side cafes and restaurants and markets geared specifically for tourists. In order to cater for the evident interesting cultural mix of people that traverse the streets of the town on a daily basis, the town has many unique non-franchised restaurants.

\section{Problem statement}

Non-franchised restaurants can be categorised as unbranded outlets with catering being either their primary or secondary activity (Eastham et al. 2001:231). Just as franchised restaurants, they require regular stock replenishments. From the data collected, via mail-and-electronic surveys and personal interviews, for non-franchised restaurants to have a competitive advantage over franchised restaurants, additional operational effort is required. Non-franchised restaurants' current practice is to utilise several suppliers. These suppliers deliver on different days in the week and at different times during the day. This practice results in setbacks: the first is that the restaurants are faced with frequent interruptions. For example, a restaurant places an order to replenish its stock. This restaurant may, based on their supplier-consumer relationship, be able to anticipate the delivery day. However, the time at which the ordered stock will be delivered is associated with huge variances and is more difficult to estimate; furthermore, since nonfranchised restaurants have numerous suppliers, this situation is aggravated. As a consequence the restaurants may experience unnecessary delays and time lost whilst awaiting deliveries. This is not only unwanted and frustrating, but may result in the loss of potential customers. Another setback may be the generation of irregular and unnecessary traffic flow. Lastly, from the data 
collected it became evident that no suppliers make use of the same logistic organisation(s) or delivery trucks, even though they may share the same restaurant(s) as customers. The current practice followed is clearly not optimal.

\section{The research proposal}

It is proposed to promote the use of a third party supplier hub that acts as a mediator between the non-franchised restaurants and their suppliers. All the suppliers will deliver to the proposed distribution centre (DC) and all the restaurants will be served from this same DC. However, because Stellenbosch has numerous non-franchised restaurants and wine estates with non-franchised restaurants, the restaurants need to be clustered. This clustering entails that the restaurants, which are positioned relatively close to one another or which have similar order types, be grouped and served together on one route assignment. (e.g. two routes will result in two clusters [that is groups] of restaurants.) The success of such a cluster installation is; however, dependent on the location of the third party supplier hub. The hub should be located such that the greatest savings in distance travelled can be achieved and that appropriate clustering can, for example, result in minimizing the traffic flow generated by the deliveries made. A route scheduling algorithm was used to help achieve this target.

\section{Literature review}

\section{Third party supplier hub}

A supplier hub is a DC with its location sited near a manufacturer's facility with all or some of its supplies stored with the general agreement that the ordered materials will be supplied on call and also paid for only when consumed (Shah \& Goh 2006:239-252). This supplier-buyer relationship is typical to that followed in a vendor managed inventory (VMI) model. In a VMI model, the supplier merely manages the customer's inventory at the customer's DC or at a manufacturing node in the network. This characteristic makes the VMI model especially useful for companies with changing and/or uncertain market demand, short product life cycles, and uncertain supply lead times.

A supply hub helps to reduce both the high scope of error in forecasting, and the uncertainty imposed on suppliers to keep sufficient safety stock throughout the supply chain. If supply hubs are located optimally, the maximum measure related to transport distance or cost may be minimized (Campbell 2009:3107-3116). The purpose of a supply hub is to have a ready supply of products available to support manufacturing operations undertaken either by the client or by a contract manufacturer (CM). With this approach, the client or CM has access to a ready supply of inventory at none or very little inventory carrying costs to them (Shah \& Goh 2006:239-252).

The operation and management of a supply hub is usually handed over to a third party logistics (3PL) provider. The concept of a 3PL involves the utilisation of external organisations to perform logistical activities that were traditionally executed within the organisation itself. Nevertheless, the ownership of the inventory and the inventory management in the hub remains the supplier's responsibility. The client or the CM usually provides a minimum amount (similar to safety stock kept in certain DCs) of the inventory levels that are to be maintained at the supplier hub. This inventory level is overseen by the supplier operator and an information system, which triggers messages to the vendors whenever the inventory stocked at the hub falls below a certain minimum, can also be utilised (Shah \& Goh 2006:239-252). By monitoring the inventory levels closely, the production and delivery schedules can be coordinated. The suppliers are able to observe the actual consumption rate of the products by the client or the CM. At a later stage this information can be used as a reference in determining the accuracy of the demand projection provided by the client or the CM and may, as a result, also decrease future forecast variances (Shah \& Goh 2006:239-252).

\section{Supplier hub structure}

A supplier hub can be located in several different manners; each forming its own distinctive transport network. For the purpose of this project only a hub and spoke network was considered.

A hub and spoke (H\&S) transport network can be defined as a set of integrated nodes where some type of information or material flow is originated, terminated or communicated from a central hub. The nodes are connected by spokes (i.e. arcs) to the central hub. These spokes represent roads, routes or other channels through which transport entities may flow (Taha et al. 1996:327-346). It is important to note here that this structure only provides single coverage (Daganzo 2009:434-446). Information or material flow may only be executed from the central hub. An H\&S transport network is commonly used in less than truckload lots (LTL) and definite time motor trucking. Both these trucking methods implement consolidation transport. That is, the movement of small but economical loads whilst still providing a quality service (Taha et al. 1996:327-346). Further, due to a H\&S network's limited level of service (LOS), the usage of this network may decrease the kilometres travelled per truck driver per day (Daganzo 2009:434-446). However, if it is assumed that truck drivers work at fixed fees, this network may be disadvantageous for trucking companies. If this assumption is valid, trucking companies will spend more per kilometre travelled per truck driver per day. In a mesh network, each node in the network acts independently, regardless of whether it is connected to another network or not. It also allows for continuous connections and rearrangements around broken or blocked paths by jumping from node to node until the final destination is reached. This network permits double coverage of information or material flow (Daganzo 2009:434-446). With such a network in place, the kilometres travelled per truck driver per day may be higher than in an H\&S network due to its broader LOS. And if it is again assumed that truck drivers work at fixed fees, 
a mesh network may result in a smaller cost per kilometre travelled per truck driver per day. However, Daganzo (2009:434-446) states that a mesh network, in comparison to an H\&S network, is more expensive to build and to operate. If one considers the characteristics of both these networks, the concept of a hybrid H\&S network is stimulated. A hybrid H\&S network has the structure of an H\&S network, but with some direct routes incorporated within the design of the network. This type of network permits for single as well as double coverage. Moreover, a hybrid H\&S network combines the advantages of both networks and results in a more cost effective network with medium LOS that is intuitive and easy to operate (Daganzo 2009:434-446).

\section{Cluster analysis}

A cluster is a subset of a set of objects with objects in the subset in some sense closer to one another than to the other objects in the set (Banerjee \& Rosenfeld 1992:963-974). Cluster analysis is the process of meaningfully grouping these objects based on a set of attributes or features in order to form an optimal subset. The purpose of clustering is to analyse and shape real world phenomena and to obtain and preserve knowledge by relating different aspects of the phenomena with one another (Budayan et al. 2009:11 772-11 781). Clustering is commonly used with inventory management. Inventory management involves keeping track of hundreds of items spread across multiple locations with complex interrelationships between them (Srinivasan \& Moon 1999:615-633). As an example, consider managing stock keeping units (SKUs) through their journey in the supply chain network. Evidently, it would not be computationally feasible to consider each product individually during the decision making process. The use of groups of items provides management with more effective methods for characterising and controlling system performance and also results in cost savings such as group discounts (Srinivasan \& Moon 1999:615-633). Another possible application of clustering is to combine the clustering concept with that of a third party supplier hub. This combination may result in a cluster of optimally located customers that are all served from the central hub evoked by the H\&S network. However, it is pertinent that all suppliers deliver to the central hub and not separately to all the individual customers. By using clustering within an H\&S network, the tour length travelled by the trucking companies can be reduced and the truckload driver's scheduling can be improved. A benefit of this combination is satisfied customers that receive once-off deliveries at more frequent intervals. Nevertheless, as mentioned previously, the suggested utilisation of an H\&S network might not be the most favourable solution. If a trucking company only has one truck serving the cluster of customers, the truck will have to, before travelling to the next consumer, reload its stock at the central hub. If a trucking company wants to serve all its customers simultaneously, a number of trucks representing the number of pre-determined clusters will have to be in place. If a hybrid H\&S model is however utilised, the trucking company may suffice with only one truck, since reloading at the central hub is not a necessity. The advantages and disadvantages of these two possible options will have to be weighed according to the specific scenario for which it is to be used.

\section{Research design}

The aim of this article was to elucidate the need for the provision of a hub-configured supply chain that services nonfranchised restaurants. The relating implementation steps and benefits of such a hub structure were researched. It was set out to determine the appropriate restaurant clusters and then to determine the best order for serving these restaurants. The emphasis was placed on executing deliveries in a group fashion by clustering the non-franchised restaurants based on their closeness to one another. The objective is that a cluster of restaurants be served on one route assignment with each route serving a particular group of restaurants. In order to achieve optimal clustering, the third party supplier hub needed to be located in such a way that (1) less fuel will be consumed, (2) less pollution will be emitted, (3) less traffic will be generated and (4) less operating expenses will be experienced by the suppliers. The Clark and Wright's savings algorithm was identified as the means to achieve this target. Unlike many vehicle routing algorithms, this algorithm caters for both the routing and the scheduling calculation. It allows for the restaurants to be clustered into appropriate groups (according to the nearest-neighbour concept), it determines the order in which the restaurants should be visited along each route, and it also incorporates the capacity limit of the delivery trucks utilised.

\section{The vehicle routing problem}

In 1964 Clarke and Wright developed an algorithm that provides a solution to the classical vehicle routing problem (VRP). This algorithm is based on the so-called savings concept and is characterised as follows (Lysgaard 1997:1):

- Goods should be delivered in given quantities to given customers from a specific central hub.

- A number of vehicles, each with a certain capacity with regard to the quantities demanded, are available for the transport of the goods.

- Every vehicle that is applied in the solution should cover a route, starting and ending at the hub, on which the goods are delivered to one or more of the customers.

The difficulty in this problem lies in determining (1) the allocation of the customers along the routes (that is the clustering of the restaurants), (2) the order in which the customers should be visited on a route, and (3) the vehicle that should cover a route. The objective is to find a solution that minimizes the total transport cost. The transport cost is specified as the cost of driving from any point to any other point, where the point may be seen as a node in the network considered. (These transport costs are not necessarily identical in the two directions applicable to the arc between the two given points. This may be due to the difference in the contour or traffic patterns leading to unidirectional 
congestions. However, this was not taken into account in this article.) Additional criteria entail that the solution satisfies the requirement that every customer is visited exactly once, that the demanded quantities are delivered orderly, and that the total demand on every route does not exceed the vehicle's capacity.

\section{The savings algorithm}

The savings algorithm is a heuristic algorithm and therefore does not always provide an optimal solution to the problem. The algorithm does, however, often yield a solution that deviates little from the optimal solution.

The basic savings concept expresses the cost savings obtained by combining two routes into only one route. This is illustrated in Figure 1 (Lysgaard 1997:2) in which point 0 represents the hub.

In scenario (a) of Figure 1, customers $i$ and $j$ are visited on separate routes. A possible alternative to this is to visit the two customers on the same route, for example in the sequence $i-j$. This is illustrated in scenario (b). If the different transport costs are known, the savings that result from driving the route in scenario (b) instead of the two routes in scenario (a) can be calculated. If the transport cost between two given points $i$ and $j$ are given by $C_{i j^{\prime}}$ the total transport cost $D_{a}$ of scenario (a) follows in Equation 1 (Lysgaard 1997:3).

\section{Equation 1: Transport cost for scenario (a)}

$D_{a}=C_{0 i}+C_{i 0}+C_{0 j}+C_{j 0}$

The transport cost $D_{b}$ of scenario (b) can be calculated similarly. Refer to Equation 2 (Lysgaard 1997:3).

\section{Equation 2: Transport cost for scenario (b)}

$D_{b}=C_{0 i}+C_{i j}+C_{j 0}$

By computing the difference between these two scenarios, the corresponding savings $S_{i j}$ can be obtained. Intuitively, relatively large values of $S_{i j}$ indicate that it is attractive, with regard to transport cost, to visit points $i$ and $j$ on the same route such that point $j$ is visited immediately after point $i$. The total savings $S_{i j}$ is shown in Equation 3 (Lysgaard 1997:3).

\section{Equation 3: Savings acquired when using scenario (b) over scenario (a)}

$S_{i j}=D_{a}-D_{b}=C_{i 0}+C_{0 j}-C_{i j}$

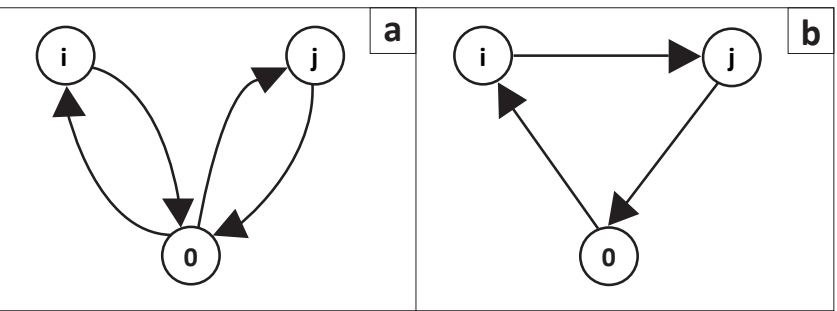

FIGURE 1: Illustration of the savings concept.

\section{Method followed in the savings algorithm}

Lysgaard (1997:5) defines the method followed in the savings algorithm as follows: in the first step of the savings algorithm, the savings for all pairs of customers are calculated and then sorted in descending order according to the savings acquired. This forms the savings list. Then, from the top of this list of node pairs, one pair of nodes is considered at a time. When a pair of nodes $(i-j)$ is considered, the two routes that visit $i$ and $j$ are combined such that $j$ is visited immediately after $i$ on the resulting route. This combination is only valid if it can be accomplished without removing a previously established direct connection between two nodes, and if the total demand on the resulting route does not exceed the vehicle's capacity. Two versions of the savings algorithm exist: a sequential and a parallel version. In the sequential version one starts anew from the top of the savings list every time a connection is established between a pair of nodes. The reason for this is that combinations, that were not viable so far, could maybe have become viable. This results in building exactly one route at a time, whilst the routes with only one customer are excluded. In the parallel version, only one pass through the list is required, since more than one route may be built at a time. According to Lysgaard (1997:5), the parallel savings algorithm frequently provides better results than the sequential savings algorithm. As a result, even though the parallel version of the Clark and Wright's savings algorithm may involve greater computational effort, it was utilised in this article.

\section{Research methodology}

In order to investigate the benefits of providing a hubconfigured supply chain, a systematic implementation approach was used. The first step in the realisation of such a hub was to do market research in order to establish the need for and the viability of a hub from a restaurant's perspective. The next step entailed the investigation of the design criteria needed in determining a favourable hub location. Four possible hub locations were identified. The Clark and Wright's savings algorithm was then programmed in Microsoft Excel to determine the most optimal hub location and the feasibility of the results was verified with the aid of a global positioning system (GPS) device.

\section{Market research}

In order to gain insight into the factors influencing a hubtype decision, data were collected via mail-and-electronic surveys and personal interviews. A statistical analysis was then executed. A population size of 73 restaurants was considered from which a random sample of 25 restaurants was selected. A discussion of the main results obtained and the corresponding remarks and implications follows:

- The peak operating months are from November to February: this period correlates with the period when the students are on holiday. This indicates that the restaurants' target market is in fact the Stellenbosch residents (and tourists) 
and not the students. Given this finding, the hub will have to make provision for the increase in demand over this period by planning for high demand scenarios.

- The peak time in the peak and off-peak period is in the afternoon: this peak time needs to be taken into account. The hub should strive to do deliveries in the mornings.

- The peak days in the peak and off-peak period are over weekends: These peak days need to be taken into account. The delivery schedules practiced by the hub need to ensure that sufficient products are delivered prior to the weekend.

- The restaurants mostly have between 10 and 20 different suppliers: this number fluctuates between smaller and larger restaurants with smaller restaurants having fewer suppliers and vice versa. In addition, this number is also influenced by whether a restaurant is positioned in-town, slightly out of town, or on a wine estate.

- The frequency of the deliveries fluctuates between once per day, more than once per day, every two days and in severe cases every three days: the smaller restaurants prefer more frequent and smaller deliveries due to the limited space in their kitchen areas. Nonetheless, except for the smaller restaurants' capacity constraints, all of the restaurants use perishable goods that need to be supplied on a regular basis as well as popular products that need regular replenishment.

- The delivery day (regardless if it is the peak or off-peak period) preferred for replenishing stock for the week is a Monday and for the weekend a Friday: a sufficient number of restaurants would prefer that deliveries also be made on a Sunday. This would enable them to finish each week's stocktaking prior to the start of the next week. This should be taken into account in managing and operating the proposed hub.

- A reaction time of a maximum of three days is classified as good service: the restaurants are, in general, satisfied with their suppliers' products and services. Further, if inaccuracies do occur, the restaurants (especially those that are positioned in central Stellenbosch) can easily make the necessary rectifications by merely buying whatever is lacking and needed at their nearest food store.

The aforementioned discussion on the results obtained and the corresponding remarks and implications relate to the operational side of the proposed third party supplier hub. However, for the application of this article, the core aspect investigated in these surveys relates to whether: once-off deliveries (combining the products of all suppliers) made from a central hub would be preferred over current delivery practices?

It was found that the restaurants are not familiar with the concept of a proposed hub structure and they, accordingly, exhibit reluctance to change. The restaurants are accustomed to and prefer the one-to-one interaction with the different suppliers, since this aids them in knowing and getting to know each supplier's expertise. The main deterrent in the realisation of a hub-configured supply chain is thus to convince the restaurants to relinquish their supplier-basis and start anew. Moreover, it was found that the restaurants on the wine estates are predominantly in favour of the proposed concept. Because these restaurants tend to be positioned further apart from one another, the advantages that they would reap cannot be overlooked. In comparison to the other restaurants, which are generally located closer to one another, the restaurants on the wine estates may experience even greater distance savings by utilising the recommended cluster installation and route scheduling.

\section{Location of the proposed hub}

In order to determine the optimal position of the hub, four industrial sites in the Stellenbosch area were identified. The proposed locations are: Techno Park, Devon Valley, Koelenhof Station and Plankenbrug. These hub locations were chosen by utilising Google Earth and then seeking possible open areas. It was assumed that initially no consent from any land owner is needed. With each of these specific locations the coordinates at its centre point were determined and the latitudes and longitudes thereof can be seen in Table 1 .

\section{Vehicle routing problem excel solution}

The Clark and Wright's saving algorithm was utilised to determine the most optimal hub location. This route scheduling algorithm was programmed in Microsoft Excel by using the language, Visual Basic. As mentioned previously, the objective of this algorithm is to find a solution that minimizes the total transport cost. This solution also satisfies the criteria that every restaurant is visited exactly once and that the total demand on every route does not exceed the truck's capacity. The 73 non-franchised restaurants were considered in showing the potency of the proposition made herein. By using the longitudes and latitudes of these restaurants, the Clark and Wright's savings algorithm determined the manner in which the restaurants should be allocated amongst the routes (that is the clustering) and the order in which the restaurants should be visited on each route. The total transport cost for all the routes, and for each proposed hub location, was calculated with the aid of the great circle method and an appropriate distance matrix. The results were then evaluated based on the hub position that resulted in the largest distance savings. And then, from these distance savings, the optimal location was evident.

\section{The use of a global positioning system}

A test with a local Garmin Nuovu GPS model TT2 was set out in order to verify the results obtained in the previously mentioned algorithmic approach. This verification was executed by driving the determined routes and by using the standard function of the GPS software to choose the shortest route. This execution method improves upon the

TABLE 1: Co-ordinates of the proposed hub locations.

\begin{tabular}{llll}
\hline Number & Hub Location & Latitude & Longitude \\
\hline 1 & Techno Park & -33.9680 & 18.8459 \\
2 & Devon Valley & -33.9463 & 18.8187 \\
3 & Koelenhof Station & -33.8788 & 18.8061 \\
4 & Plankenbrug & -33.9294 & 18.8505 \\
\hline
\end{tabular}


shortage of the algorithmic approach by providing for oneway streets and any other form of detour or deviation from the normal street or route. The results recorded confirmed, within appropriate margins, to the results found with the algorithmic approach where the great circle distance was used.

\section{Results and conclusions}

After the four promising hub locations were investigated and weighed against each other, it was determined that the most optimal hub location would be at Plankenbrug.

Based on the results in Table 2, it is apparent that the best hub location is at hub 4 (Plankenbrug) with hub 2 (Devon Valley) being the best next solution. However, since the total distance travelled to visit all the restaurants when the hub is positioned at either hub 4 (Plankenbrug) or hub 2 (Devon Valley), it can be argued that either one of them will result in sufficient fuel cost savings. Further, since both these hub locations utilise only seven routes and since the number of routes may relate to the number of trucks required by the hub (that is one truck per route), these two hub locations will both result in the hub spending less money on its operating expenses (that is transport costs). It is proposed that the deciding criterion between these two hub locations be based on the consent needed from the respective land owners. By utilising Plankenbrug over the other proposed hub locations, a distance of up to $125 \mathrm{~km}$ may be saved. If the hub is located at Plankenbrug, it was determined that the total distance (that is the distance travelled to visit all the restaurants considered herein) travelled is $150 \mathrm{~km}$. This is up to $125 \mathrm{~km}$ less than the total distance travelled when the hub was positioned at the other three proposed hub locations. This emphasises the value of clustering the restaurants and optimising the routes. In addition, it was determined that seven routes (with their origins at Plankenbrug) needed to be employed in order to visit all the considered non-franchised restaurants. The results were verified with the use of a GPS device. This verification showed that, even though the algorithm used is somewhat optimistic or tends to measure as the crow flies, the approach is still deemed suitable. In comparison to the GPS approach which involves equipment cost and travelling expenses, the algorithmic approach still showed a high level of acceptability and can be used in developing a solution for a hub configuration. Lastly, the analysis of the hub's capital and operating expenses clearly indicated the hub's profitability. It was calculated that the hub will generate a profit of R270 185 per month. This profit is after the operating expenses and capital redemption costs (over a period of 10

TABLE 2: Distances travelled.

\begin{tabular}{lll}
\hline Hub location & Total distance travelled & Number of routes required \\
\hline 1 (Techno Park) & $\pm 190 \mathrm{~km}$ & 08 \\
2 (Devon Valley) & $\pm 155 \mathrm{~km}$ & 07 \\
3 (Koelenhof St) & $\pm 275 \mathrm{~km}$ & 09 \\
4 (Plankenbrug) & $\pm 150 \mathrm{~km}$ & 07 \\
\hline
\end{tabular}

years with a rate of $15 \%$ ) are subtracted, working on a mere $8 \%$ profit margin.

\section{Selling the proposed solution}

In order to make an informed decision, the advantages and disadvantages of both the restaurants' current practice and of this article's proposed solution needed to be considered. Three viewpoints were identified: the restaurants', the suppliers' and the general public's.

If non-franchised restaurants convert to using a third party supplier hub, they will experience the following advantages. Firstly, the restaurants will receive once-off and frequent deliveries made on days and times of the day determined by them. Secondly, since the hub will buy food in bulk whilst also implementing appropriate scheduling of restaurants with trustworthy delivery policies, the restaurants will, as a result, receive high quality products at lower costs exactly when they need it. Thirdly, by partaking in this hub proposition, the restaurants will undoubtedly improve their market competitiveness. That is, due to the hub's lower selling prices, the restaurants will be able to buy and provide a broader range of products. The advantages that the suppliers will experience are that they will have regular guaranteed order quantities as well as lower overhead costs. By utilising appropriate groupings and scheduling of the restaurants less fuel will be consumed (that is less fossil fuels will be emitted) and less road traffic will be generated. This will not only be advantageous to the general public, but also to the environment.

Aside from these advantages, there are; however, also two evident disadvantages. Firstly, people, in general, are resistant to change. In changing their delivery practices, the restaurants and suppliers will enter unknown territory, and that will likely be perceived as a risk to their businesses. Nevertheless, any such risks can be controlled and rectified with appropriate change management techniques. Secondly, the suppliers and restaurants that have an intimate relationship with each other may be fearful of handing their knowledge to unknown new third party suppliers. Fear of change and ownership of information are two basic human traits that will need to be managed if outlet owners are to be persuaded to embrace a major change in the servicing of their businesses. The potential benefits to be garnered are significant, not only to the individual restaurant owner but also to the wider industry and to the South African public at large. An efficient system of delivery will not only reduce disruption and costs, but will result in far lower traffic volumes. The benefits will also increase as time passes and as the people become acquainted and comfortable with the new principles and trading methods related to this proposition. In essence, it is believed that the establishment of such a hub-configured supply chain will promote sustainable development and will provide South Africa (SA) with the 
necessary impetus to grow and improve its general socioeconomic standards.

\section{Conclusions and further research}

Firstly, it is proposed that the focus of this research be expanded to restaurants on wine estates. As mentioned previously, these restaurants are positioned further from one another and therefore appropriate scheduling will result in even greater transport cost savings. These restaurants also appear to be enthusiastic about the development of a third party supplier hub. Should the results be positive and then applied to all outlying restaurants per large cities in SA, the economic impact on roads, fuel consumption, traffic congestion, tyres, repairs, maintenance, and many peripheral wastage could make a major contribution to the national benefit.

Secondly, it is proposed that the effect of operating an e-hub system, utilising e-commerce practices, be researched. This might entail the implementation of appropriate information systems. As a result, all the clustering entities (the restaurants, their suppliers and the third party supplier hub) will have access to this database. The database may facilitate the restaurants with ordering products conveniently, and the hub with efficiently and effectively scheduling their deliveries, and lead to additional savings not covered by this initial research. In conclusion, the concept of hubs and clustering is in its infancy and many positive outcomes await those that embark on this path. South Africa offers the entrepreneur many opportunities in the supply chain arena as they are identified and developed.

\section{Acknowledgements Competing interests}

The authors declare that they have no financial or personal relationship(s) that may have inappropriately influenced them in writing this article.

\section{Author contributions}

G.A.R. (University of Stellenbosch) was the project leader, C.B.S. (University of Stellenbosch) was responsible for experimental and project design and performed most of the experiments. K.V.L. (University of Stellenbosch) made conceptual contributions.

\section{References}

Banerjee, S. \& Rosenfeld, A., 1992, 'Model Based Cluster Analysis', Centre for Automation Research, University of Maryland.

Budayan, C., Dikmen, I. \& Birgonul, T., 2009, 'Comparing the Performance of Traditional Cluster Analysis, Self-organizing Maps and Fuzzy C-means method for Strategic Grouping', Dept. of Civil Engineering, Middle East Technical University.

Campbell, J.F., 2009, 'Hub Location for Time Definite Transport', College of Business Administration, University of Missouri.

Community Connections Online, 2011, 'Stellenbosch - Population, Climate and Geography', viewed 05 April 2013, from http://www.stellenboschconnect.co.za/ stellenbosch/population-climate-geography

Daganzo, C.F., 2009, 'Structure of Competitive Transit Networks', Institute of Transport Studies, University of California.

Eastham, J.F., Sharples, L. \& Ball, S. (eds.), 2001, Food supply chain management, Butterworth Heinemann.

Lysgaard, J., 1997, 'The Clark and Wright's Savings Algorithm', Dept. of Management Science and Logistics, The Aarhus School of Business.

Shah, J. \& Goh, M., 2006, 'Setting operating policies for supply hubs', International Journal of Production Economies: Elsevier 100(2), 239-252. http://dx.doi. org/10.1016/j.ijpe.2004.11.008

Srinivasan, M. \& Moon, Y.B., 1999, 'A comprehensive Clustering Algorithm for Strategic Analysis of Supply Chain Networks', Dept. of Mechanical, Aerospace and Manufacturing Engineering, University of Syracuse.

Taha, T.T., Taylor, G.D. \& Taha, H.A., 1996, 'Simulation based software system for evaluating hub and spoke transport networks', Simulation Practice and Theory (3)6, 327-346. http://dx.doi.org/10.1016/0928-4869(95)00016-X 